\title{
Effects of 17 $\beta$-Estradiol on Cognitive Performance of Ovariectomized Female Rats Exposed to Space Radiation
}

\author{
Bernard M. Rabin ${ }^{1 *}$, Kirsty L. Carrihill-Knoll ${ }^{1}$, Lauren V. Long ${ }^{1}$, \\ Steven C. Pitts ${ }^{1}$, Barbara Shukitt-Hale ${ }^{2}$ \\ ${ }^{1}$ Department of Psychology, University of Maryland, Baltimore County, Baltimore, USA \\ ${ }^{2}$ Human Nutrition Research Center on Aging, USDA-ARS, Tufts University, Boston, USA \\ Email: *rabin@umbc.edu
}

Received December 14, 2012; revised January 15, 2013; accepted January 22, 2013

\begin{abstract}
On exploratory class missions to other planets astronauts will be exposed to types and doses of radiation that are not experienced in low earth orbit. While it is likely that the crew will consist of both male and female astronauts, there has been little research on the effects of exposure to space radiation on central nervous system function and cognitive performance in female subjects. Because estrogen can function as a neuroprotectant, the present experiments were designed to evaluate whether or not the presence or absence of estrogen at the time of irradiation would affect the susceptibility to the neurocognitive effects of exposure to ${ }^{56} \mathrm{Fe}$ particles in female rats. Capsules containing $17 \beta$-estradiol or vehicle were implanted in ovariectomized rats three days prior to exposure ${ }^{56} \mathrm{Fe}$ particles (50 - $200 \mathrm{cGy}, 1000 \mathrm{MeV} / \mathrm{n}$ ). Cognitive performance was evaluated using novel object recognition memory to measure learning and memory and operant responding on an ascending fixed-ratio schedule to measure changes in motivation and in the responsiveness to environmental contingencies. The results indicated the estrogen does not function as a neuroprotectant to minimize the cognitive effects of exposure to ${ }^{56} \mathrm{Fe}$ particles. However, the presence/absence of estrogen at the time of irradiation could modulate the responsiveness of the subject to the disruptive effects of exposure to HZE particles on the performance of specific cognitive tasks.
\end{abstract}

Keywords: Cosmic Rays; Behavior; Sex Differences; Estrogen

\section{Introduction}

On exploratory class missions to other planets astronauts will be exposed to types and doses of radiation, including particles of high energy and charge (HZE particles) such as ${ }^{56} \mathrm{Fe}$, that are not experienced in low earth orbit [1-3] where there International Space Station and space shuttle operate. While it is likely that the crew will consist of both male and female astronauts, there has been little research on the effects of exposure to HZE particles on central nervous system function and cognitive performance in female subjects. Both exposure to HZE particles [4-9] and the gonadal hormone environment [10-15] can affect neuronal function and cognitive performance. As such, it is possible that males and females may respond to HZE particle irradiation differently.

Estrogen has been reported to provide protection against the development of neurodegenerative diseases, delaying the onset and progression of Alzheimer's and Parkinson's diseases and schizophrenia [16-19]. In addition, estrogen protects against experimental ischemic

*Corresponding author. stroke [16,19], chronic inflammatory diseases [20] and dopaminergic system dysfunction following the administration of a variety of neurotoxins [21,22], including kainic acid and 6-hydroxydopamine.

The mechanisms underlying the neuroprotective effects of estrogen are not certain. It has been suggested that estrogen may function to activate free radical scavenging systems [23], reducing oxidative stress and minimizing neuroinflammatory processes in the brain $[24,25]$. Alternatively, the neuroprotective effects of estrogen may possibly be mediated by actions on microglia [23-26], where estrogen can affect both apoptotic [27] and kinase signaling processes $[28,29]$.

Because exposure to HZE particles produces many of the same changes in neuronal function that characterize neurodegenerative diseases, including oxidative stress [7, 30], inflammation [31], and changes in dopaminergic function [32-34], it is possible that estrogen may serve similar neuroprotective functions following exposure to HZE particles as it does for neurodegenerative diseases. The present experiments were designed to evaluate whether or not the presence or absence of estrogen at the time 
of irradiation would affect susceptibility to the neurocognitive effects of exposure to ${ }^{56} \mathrm{Fe}$ particles in female rats.

\section{Experimental Procedure}

\subsection{Subjects}

The subjects were 100 ovariectomized (OVX) female Sprague-Dawley rats weighing 175 - $200 \mathrm{~g}$ obtained from Taconic Farms. All procedures were approved by the IACUCs of Brookhaven National Laboratory (BNL) and University of Maryland Baltimore County (UMBC).

\subsection{Implantation of Estradiol/Vehicle}

Implantation of estradiol or vehicle was performed at BNL. The procedure was adapted from the one detailed by Strom and colleagues [35]. Thirty $\mathrm{mm}$ segments of silastic laboratory tubing (Inner/outer diameter: 1.575/ $3.175 \mathrm{~mm}$, Dow Corning, VWR International, Buffalo Grove, IL) were filled with a solution of 180 pg $17 \beta$-estradiol $/ \mathrm{mL}$ sesame oil or sesame oil alone (vehicle). The ends of the tubing were sealed with $5 \mathrm{~mm}$ pieces of wooden applicator sticks, resulting in a vehicle- or $17 \beta$-estradiol-filled column $20 \mathrm{~mm}$ in length. Before use, the capsules were stored overnight in a vial containing sesame oil with the same concentration of $17 \beta$-estradiol or vehicle as inside the capsules.

To implant the capsules the rats were anesthetized with sodium pentobarbital ( $35 \mathrm{mg} / \mathrm{kg}$, i.p.). A $5 \mathrm{~mm}$ incision was made in the loose skin of the rat's neck, and a pocket bluntly dissected caudally in which the silastic capsule was gently installed using forceps. The capsules were implanted subcutaneously in the neck because of its abundance of loose skin, and to minimize the risk of mechanical stress on the capsule. The incision was closed by a suture. Following surgery the rats were monitored to make certain that they recovered from the anesthesia.

Strom et al. [35] have used radioimmunoassay procedures to evaluate the levels of estradiol in serum following implantation of silastic capsules containing $17 \beta$-estradiol as detailed above. They reported that this procedure resulted in a relatively stable, physiological level of estradiol in serum from the time of implantation and lasting for 4 - 5 weeks following implantation.

\subsection{Radiation}

Because serum estradiol concentrations attained with the use of silastic capsule implants are relatively constant immediately following implantation [35] the rats were irradiated after a $48 \mathrm{hr}$ recovery period. The rats were exposed to ${ }^{56} \mathrm{Fe}$ particles at the NASA Space Radiation Laboratory (NSRL) at BNL. Dosimetry was provided by the staff of the NSRL using ionization chambers. The doses to which the rats were exposed were $50(\mathrm{n}=20)$, $100(n=24), 150(n=24)$ and $200(n=20)$ cGy at a nominal dose rate of $25-100 \mathrm{cGy} / \mathrm{min}$. The control rats (0 cGy, $n=22$ ) were taken to the NSRL but were not irradiated. Half the rats at each dose were implanted with silastic tubing containing $17 \beta$-estradiol and half were implanted with tubing containing vehicle.

For irradiation, the rats were placed in well-ventilated plastic tubes which were placed perpendicular to the beam. The animal was positioned with the center of its head in the beam. As such, some of the neck of the animal was also exposed. Following irradiation the rats were shipped to UMBC for behavioral testing.

\subsection{Behavioral Testing}

At UMBC the rats were given 7 - 10 days to recover from the effects of shipping before beginning behavioral testing. The behavioral procedures have been detailed in previous publications, so they will only be briefly described here.

\subsubsection{Object Recognition [36]}

Subjects were tested in a dimly lit open field $(93 \mathrm{~cm} \times 93$ $\mathrm{cm}$ ). The stimulus objects (which are no smaller than the size of the rat and no larger than two and a half times its size) vary in shape and color. After habituation to the apparatus, two identical (familiar) stimulus objects are placed in symmetrical locations in the open field. The rat is allowed to explore the stimuli until it accumulates 30 sec total object exploration (i.e., exploration of either object) or until 20 min have passed. After $24 \mathrm{hr}$ delay, the rat is placed back in the field with one familiar and one novel object and allowed to explore both stimuli until it has accumulated $30 \mathrm{sec}$ of object exploration on either object or until 20 min have passed. Normally rats will spend more time exploring the novel object; old rats and rats that have impaired recognition memory spend equal amounts of time with both the familiar and novel object.

\subsubsection{Operant Responding [37]}

Food-deprived rats (maintained at $90 \%$ base weight) are first trained to press a lever on a continuous reinforcement schedule using an autoshaping procedure in which they are placed in an operant chamber for 12 hours. Most rats learn the response within a single session. The rats are then trained to respond on a fixed-ratio (FR) schedule by placing them in the chamber for 30 min and rewarding them on FR-1, FR-5 (every fifth response rewarded), FR-10, and FR-20 reinforcement schedules. For testing they are then given 30-min sessions in which they are rewarded on FR-1, FR-5, FR-10, FR-15, FR-20, FR-25, FR-30 and FR-35 reinforcement schedules on consecutive days. This task is a measure of an organism's moti- 
vation to work for reinforcement and its ability to respond to changes in environmental contingencies. Performance on this task is dependent on the dopaminergic system [38] and may be related to the activational aspects of motivation and the effort put into obtaining reinforcement and is dependent upon the integrity of the striatum $[39,40]$.

\subsection{Statistics}

The initial data analyses were performed using 2-way or 3 -way analyses of variance. Comparisons between groups exposed to different doses of ${ }^{56} \mathrm{Fe}$ particles were made using Fisher's protected-T.

\section{Results}

\subsection{Novel Object Recognition}

Exposing OVX female rats, with either estradiol or vehicle implants at the time of irradiation, to ${ }^{56} \mathrm{Fe}$ particles did not affect the response to the novel object (Figure 1). All rats spent significantly more time with the novel object than with the familiar object. Neither the main effect for dose, $(\mathrm{F}[4,75]=2.40, p>0.05)$, for treatment (estradiol/vehicle) $(\mathrm{F}[1,45]=0.05, p>0.10)$, nor the dose by treatment interaction $(\mathrm{F}[4,84]=1.30, p>0.10)$ was significant.

\subsection{Operant Responding}

The effect of exposure to ${ }^{56} \mathrm{Fe}$ particles on operant responding on an ascending FR schedule in OVX female rats is shown in Figure 2. A 3-way ANOVA with one

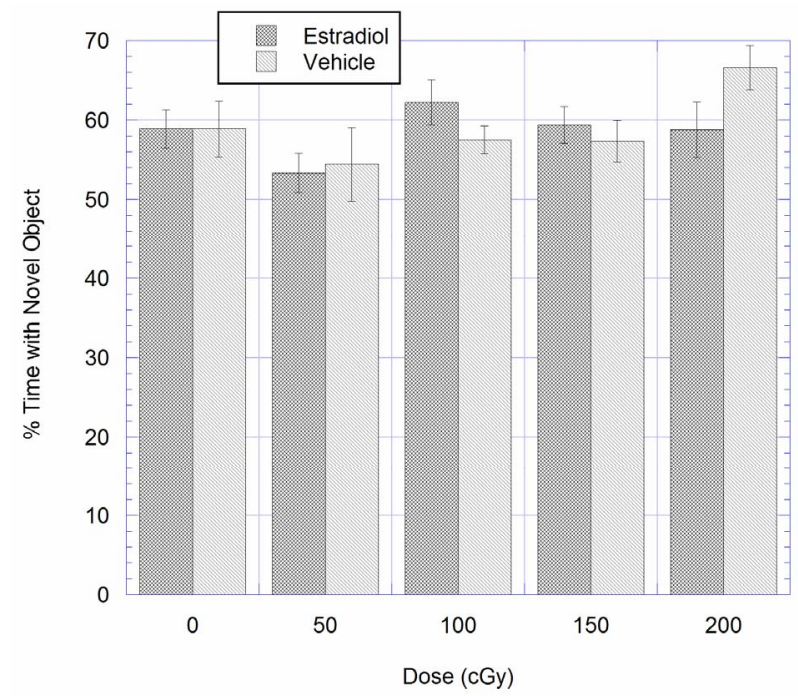

Figure 1. The percentage of time spent exploring the novel or familiar object in female rats implanted with $17 \beta$ estradiol or vehicle and irradiated with ${ }^{56} \mathrm{Fe}$ particles. Mean \pm standard error of the mean (s.e.m.). repeated variable (reinforcement schedule) was used for the initial analysis. The ANOVA indicated that the main effects for dose, treatment (estradiol or vehicle implant) and reinforcement schedule were all significant (F[4,90] $=2.54, p<0.05 ; \mathrm{F}[1,90]=4.08, p<0.05 ; \mathrm{F}[7,630]=$ $39.70, p<0.01$; respectively). The dose by schedule interaction was significant $(\mathrm{F}[28,630]=1.50, p<0.05)$ as was the treatment by schedule interaction $(\mathrm{F}[7,630]=$ 2.77, $p<0.01$ ]. Neither the dose by treatment interaction nor the triple interaction was significant.

To partial out the main effects, two different 2-way

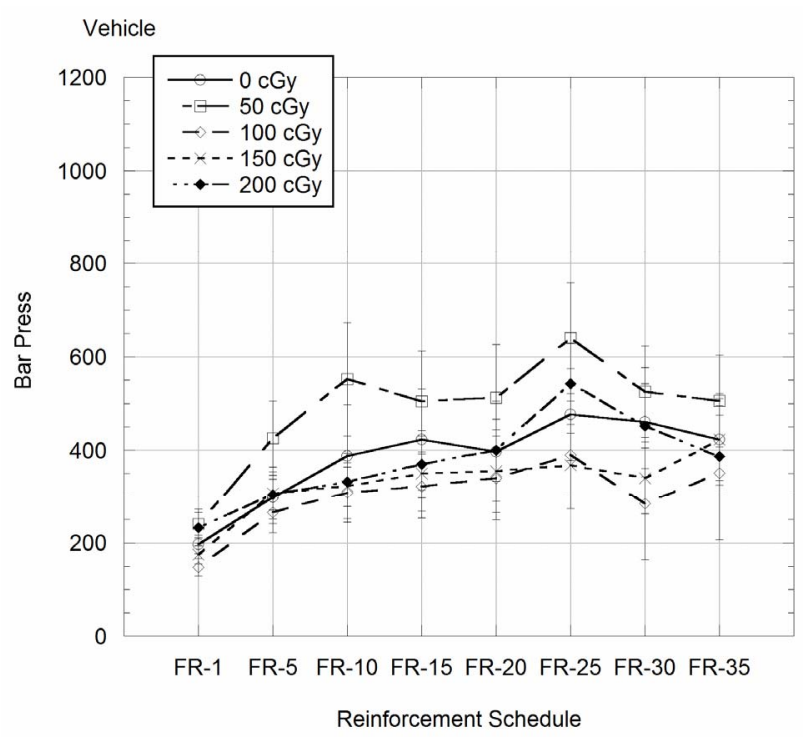

(a)

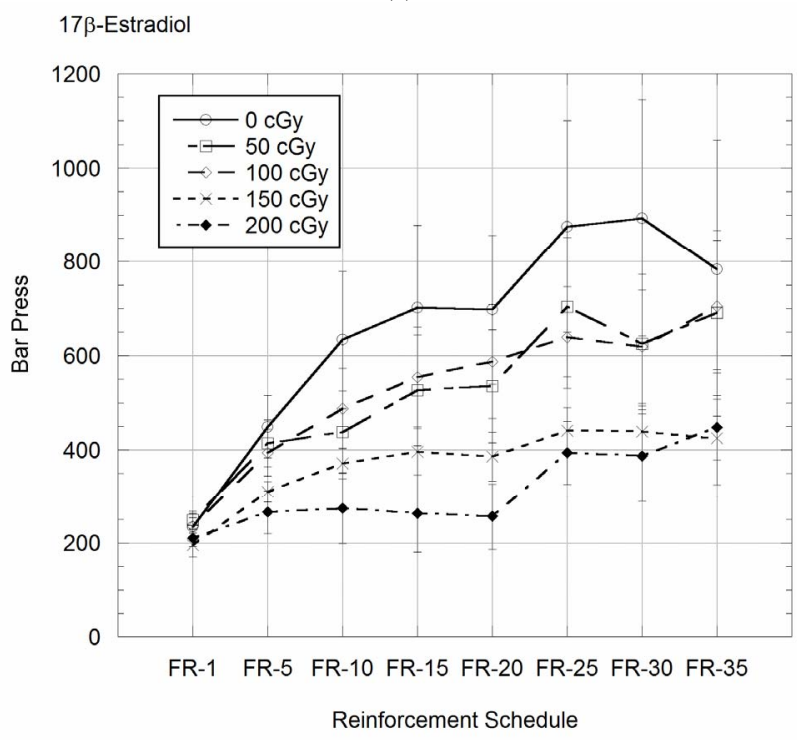

(b)

Figure 2. Performance on an ascending fixed-ration operant task in female rats exposed to ${ }^{56} \mathrm{Fe}$ particles (0 - $\left.200 \mathrm{cGy}\right)$. Upper panel: capsules containing vehicle only implanted prior to irradiation; lower panel: capsules containing estradiol $17 \beta$ implanted. Mean \pm s.e.m. 
ANOVAs were run for each of the implant conditions using the MS error from the 3-way ANOVA [41]. For the animals given estradiol implants, the main effect for dose of radiation $(\mathrm{F}[4,90]=2.77, p<0.05)$ and for reinforcement schedule $(\mathrm{F}[7,28]=17.63, p<0.01)$ were significant. The dose by schedule interaction was also significant $(\mathrm{F}[28,630]=1.62, p<0.01)$ indicating that performance deteriorated as a greater number of responses were needed to produce reinforcement. In contrast, for the rats given vehicle implants, only the main effect for reinforcement schedule was significant. Neither the main effect of dose nor the dose by schedule interactions was significant.

Because visual inspection of Figure 2 suggests the possibility that there were differences in responding in the non-irradiated control animals as a function of estradiol or vehicle implants, a final 2-way ANOVA was run to compare the performance of the non-irradiated animals (0 cGy) given either estradiol or vehicle implants. For both treatment groups (estradiol or vehicle) there was a significant increase in response rate as the reinforcement schedule increased $(\mathrm{F}[7,140]=6.95, p<0.01)$. However, there were no differences in performance as a function of treatment condition $(\mathrm{F}[1,20]=1.78, p>0.10)$, or in the treatment by schedule interaction $(\mathrm{F}[7,175]=0.98, p>$ $0.10)$. These results indicate that the differences in responding between animals given estradiol or vehicle at the time of radiation could not be due to differences in hormonal status only, but must reflect an interaction between hormonal status and exposure to ${ }^{56} \mathrm{Fe}$ particle.

\section{Discussion}

While the results suggest that estrogen may exert both organizational and activational effects on cognitive performance following exposure to HZE particles, depending upon the specific task, overall, the results do not support the original hypothesis that estrogen can function as a neuroprotectant to counteract the effects of exposure to ${ }^{56} \mathrm{Fe}$ particles on cognitive performance. Rather, the presence of estrogen at the time of irradiation seems to be necessary for the particle-induced disruption of operant responding. In addition, although male subjects were not run at the present time, comparison with the results of previous experiments utilizing male rats [36,37] suggests that male and female rats may respond differently to exposure to HZE particles, depending upon the specific task.

In contrast to operant performance none of the OVX rats, given either estradiol or vehicle at the time of irradiation, showed a disruption of recognition memory following exposure to ${ }^{56} \mathrm{Fe}$ particles. All rats, both radiated and non-irradiated controls, spent more time interacting with the novel object than with the familiar object. Al- though the hormonal environment for these rats differed at the time of irradiation, depending upon whether the rats were given estradiol or vehicle implants, there were no differences in the hormonal environment at the time of testing because the estradiol levels measured in blood would have returned to normal OVX levels by the time they were tested on the novel object recognition task which occurred 4 - 6 weeks following surgery [35]. As such, these results indicate that the hormonal environment (presence or absence of estradiol) at the time of irradiation was not a factor influencing the effects of exposure to ${ }^{56} \mathrm{Fe}$ particles on recognition memory in female rats. These results differ from those obtained using male subjects which show a disruption of recognition memory following exposure to ${ }^{56} \mathrm{Fe}$ particles [36] such that equal amounts of time are spent with both novel and familiar objects. The failure to observe a radiation-induced performance decrement in female rats, compared to male rats, following exposure to ${ }^{56} \mathrm{Fe}$ particles may reflect differences in hormone levels at the time of testing or in the organization of the brain as the result of differences in perinatal hormones [42]. Alternatively, the differences between males and females may reflect the fact that gonadal hormones have can have different effects on cognitive performance in males and female [43-45].

Operant responding on an ascending FR schedule, a measure of the responsiveness of the organism to environmental contingencies, showed distinct changes in the patterns of responding as a function of hormonal status at the time of irradiation. The ovariectomized females given vehicle-only implants showed no effect of exposure to ${ }^{56} \mathrm{Fe}$ particles on their response pattern: there were no differences in response patterns between the non-irradiated control rats and the irradiated rats. However, the OVX females given estradiol implants showed an effect of irradiation on cognitive performance following irradiation. Compared to the non-irradiated controls, the OVX rats with estradiol implants at the time of irradiation failed to show a corresponding increase in responding as the reinforcement schedule increased following exposure to 150 or $200 \mathrm{cGy}$ of ${ }^{56} \mathrm{Fe}$ particles. In this regard, the performance of the female rats with estradiol implants at the time of irradiation was similar to that of male rats which showed a disruption of operant responding following exposure to $200 \mathrm{cGy}$ of ${ }^{56} \mathrm{Fe}$ particles [37].

The mechanisms underlying the interaction between exposure to ${ }^{56} \mathrm{Fe}$ particles and estradiol in OVX rats which result in the disruption of operant responding are not certain. Performance on this specific task is dependent upon the integrity of the dopaminergic system [38]. Gender differences have been reported in the functioning of the dopaminergic system in the striatum, with estrogen enhancing the release of dopamine in female, but not male rats [46]. Estrogen has been reported to have dif- 
ferent effects on striatal- and hippocampal-mediated learning [10], such that estrogen facilitates hippocampal-mediated learning while it interferes with striatally-mediated learning. It has been further suggested that the interaction between estrogen and dopamine influences the use of striatally- or hippocampally-mediated strategies in the performance of a cognitive task [13].

Similar task-dependent sex differences following exposure to HZE particles have been reported by Villasana et al. [8]. Exposure to ${ }^{56} \mathrm{Fe}$ particles impaired performance of hippocampal-dependent contextual fear conditioning task in female mice, but led to improved performance in male mice. There was no effect of exposure to ${ }^{56} \mathrm{Fe}$ particles in the performance of a cued fear conditioning task, which is not mediated by the hippocampus, in either male or female mice.

Overall, the results of these experiments indicate that the relationship between the gonadal hormone environment and cognitive performance following exposure to ${ }^{56} \mathrm{Fe}$ particles is complex. The effects of gonadal hormones on neuronal function can be classified as "activational" or "organizational" [42,47]. Activational effects refer to contemporary correlations between the current hormonal environment and cognitive performance. Organizational effects refer to developmental correlations between the perinatal hormonal environment which influences the organization of the brain, and which may, at some later time, affect cognitive performance. Both types of effects are observed following exposure to ${ }^{56} \mathrm{Fe}$ particles. Estradiol- and vehicle-implanted rats showed no differences in performance on the novel object recognition task, indicating that the hormonal environment, presence or absence of estrogen at the time of irradiation, had no effect on subsequent performance. However, the observation that neither the estradiol or vehicle implanted females, in contrast to males [36], spent more time with the novel object following irradiation suggests that there may be differences in brain organization which affect performance on this task. In contrast, activational effects were observed in the effects of exposure to ${ }^{56} \mathrm{Fe}$ particles on operant performance.

In summary, the results of these experiments indicate that estrogen, despite its capacity to function as a neuroprotectant following a variety of toxic insults $[19,21,22]$ or neurodegenerative disorders [16,18], may not fulfill that role following exposure to HZE particles. Rather, the results suggest that the activational effects of estrogen may be to produce a performance deficit with selected cognitive tasks, specifically operant responding on an ascending fixed ratio reinforcement schedule which is a measure of the responsiveness of the organism to changes in environmental contingencies. As such, the presence of estrogen at the time of irradiation seems to function to make females respond to exposure to ${ }^{56} \mathrm{Fe}$ particles in a manner similar to males. The role of testosterone in male subjects remains to be established.

\section{Acknowledgements}

This research was supported by NASA grant NNX08 AM66G.

\section{REFERENCES}

[1] F. A. Cucinotta, W. Schimmerling, P. B. Saganti, J. W. Wilson, L. E. Peterson, G. D. Badhwar and J. F. Dicello, "Space Radiation Cancer Risks and Uncertainties for Mars Missions,” Radiation Research, Vol. 156, No. 5, 2001, pp. 682-688. doi:10.1667/0033-7587(2001)156[0682:SRCRAU]2.0.C $\mathrm{O} ; 2$

[2] A. A. Edwards, "RBE of Radiations in Space and the Implications for Space Travel,” Physica Medica, Vol. 27, 2001, pp. 147-152.

[3] W. Schimmerling, F. A. Cucinotta and J. W. Wilson, "Radiation Risk and Human Space Exploration" $A d$ vances in Space Research, Vol. 31, No. 1, 2003, pp. $27-$ 34. doi:10.1016/S0273-1177(02)00653-1

[4] G. Casadesus, B. Shukitt-Hale, H. M. Stellwagen, M. A. Smith, B. M. Rabin and J. A. Joseph, "Hippocampal Neurogenesis and PSA-NCAM Expression Following Exposure to ${ }^{56} \mathrm{Fe}$ Particles Mimics That Seen during Aging in Rats," Experimental Gerontology, Vol. 40, No. 3, 2005, pp. 249-254. doi:10.1016/j.exger.2004.09.007

[5] N. Denisova, B. Shukitt-Hale, B, M. Rabin and J. A. Joseph, "Brain Signaling and Behavioral Responses Induced by Exposure to ${ }^{56} \mathrm{Fe}$ Radiation," Radiation Research, Vol. 158, No. 6, 2002, pp. 725-734. doi:10.1667/0033-7587(2002)158[0725:BSABRI]2.0.CO; $\underline{2}$

[6] J. A. Joseph, B. Shukitt-Hale, J. McEwen and B. M. Rabin, "CNS-Induced Deficits of Heavy Particle Irradiation in Space: The Aging Connection,” Advances in Space Research, Vol. 25, No. 10, 2000, pp. 2057-2064. doi:10.1016/S0273-1177(99)01013-3

[7] B. Shukitt-Hale, G. Casadesus, A. Carey, B. M. Rabin and J. A. Joseph, "Exposure to ${ }^{56} \mathrm{Fe}$ Irradiation Accelerates Normal Brain Aging and Produces Deficits in Learning and Memory,” Advances in Space Research, Vol. 39, No. 6, 2007, pp. 1087-1092. doi:10.1016/j.asr.2006.11.005

[8] L. Villasana, J. Rosenberg and J. Raber, “Sex-Dependent Effects of ${ }^{56} \mathrm{Fe}$ Irradiation on Contextual Fear Conditioning in C57BL/6J Mice,” Hippocampus, Vol, 20, 2010, pp. 19-23.

[9] R. Vlkolinsky, T. Krucker, A. L. Smith, T. C. Lamp, G. A. Nelson and A. Obenhaus, "Effects of Lipopolysaccharide on ${ }^{56} \mathrm{Fe}$-Particle Radiation-Induced Impairment of Synaptic Plasticity in the Mouse Hippocampus," Radiation Research, Vol. 168, No. 4, 2007, pp. 462-470. doi:10.1667/RR1038.1

[10] D. M. Davis, T. K. Jacobsen, S. Aliakbari and S. J. Y. 
Mizumori, "Differential Effects of Estrogen on Hippocampal- and Stiratal-Dependent Learning," Neurobiology of Learning and Memory, Vol. 84, No. 2, 2005, pp. 132137. doi:10.1016/j.nlm.2005.06.004

[11] L. A. M. Galea, "Gonadal Hormone Modulation of Neurogenesis in the Dentate Gyrus of Adult Male and Female Rodents," Brain Research Reviews, Vol. 57, No. 2, 2008, pp. 332-341. doi:10.1016/j.brainresrev.2007.05.008

[12] J. Prange-Kiel and G. M. Rune, "Direct and Indirect Effects of Estrogen on Rat Hippocampus,” Neuroscience, Vol. 138, No. 3, 2006, pp. 756-772. doi:10.1016/j.neuroscience.2005.05.061

[13] M. G. Quinal, D. Hussain and E. G. Brake, "Use of Cognitive Strategies in Rats: The Role of Estradiol and Its Interaction with Dopamine," Hormones and Behavior, Vol. 53, No. 1, 2008, pp. 185-191. doi:10.1016/j.yhbeh.2007.09.015

[14] F. Van Haaren, A. van Hest and R. P. W. Heinsbroek, "Behavioral Differences between Male and Female Rats: Effects of Gonadal Hormones in Learning and Memory," Neuroscience and Biobehavioral Reviews, Vol. 14, No. 1, 1990, pp. 23-33. doi:10.1016/S0149-7634(05)80157-5

[15] M. Wallace, V. Luine, A. Arekkanos and M. Frankfurt, "Ovariectomized Rats Show Decreased Recognition Memory and Spine Density in the Hippocampus and Prefrontal Cortex,” Brain Research, Vol. 1176, No. 1, 2006, pp. 176-182. doi:10.1016/j.brainres.2006.07.064

[16] D. E. Brann, K. Dhandapani, C. Wakade, V. B. Mahesh and M. M. Khan, "Neurotrophic and Neuroprotective Actions of Estrogen: Basic Mechanisms and Clinical Implications,” Steroids, Vol. 72, No. 5, 2007, pp. 381-405. doi:10.1016/j.steroids.2007.02.003

[17] L. M. Garcia-Segura, I. Azcoitiaa and L. L. DonCarlos, "Neuroprotection by Estradiol," Progress in Neurobiol$o g y$, Vol. 63, No. 3, 2001, pp. 29-60. doi:10.1016/j.physbeh.2005.04.007

[18] G. E. Gilles and S. McArthur, "Independent Influences of Sex Steroids of Systemic and Central Origin in a Rat Model of Parkinson's Disease: A Contribution to SexSpecific Neuroprotection by Estrogens," Hormones and Behavior, Vol. 57, No. 1, 2010, pp. 23-34. doi:10.1016/j.yhbeh.2009.06.002

[19] P. S. Green and J. W. Simpkins, "Neuroprotective Effects of Estrogens: Potential Mechanisms of Action,” International Journal of Developmental Neuroscience, Vol. 18, No. 4-5, 2000, pp. 347-358. doi:10.1016/S0736-5748(00)00017-4

[20] L. Charalampopoulos, E. Remboutsika, A. N. Margoris and A. Gravanis, "Neurosteroids as Modulators of Neurogenesis and Neuronal Survival," Trends in Endocrinology and Metabolism, Vol. 19, No. 8, 2008, pp. 300-307. doi:10.1016/j.tem.2008.07.004

[21] D. E. Dluzen, "Estrogen Decreases Corpus Striatal Neurotoxicity in Response to 6-Hydroxydopamine," Brain Research, Vol. 767, No. 2, 1997, pp. 340-344. doi:10.1016/S0006-8993(97)00630-6

[22] D. E. Dluzen, "Neuroprotective Effects of Estrogen upon the Nigrostratal Dopaminergic System,” Journal of Neu- rocytology, Vol. 29, No. 5-6, 2000, pp. 387-399. doi:10.1023/A:1007117424491

[23] J. Nilson, "Estradiol and Neurodegenerative Oxidative Stress," Frontiers in Neuroendocrinology, Vol. 29, No. 4, 2008, pp. 463-475. doi:10.1016/j.yfrne.2007.12.005

[24] M. Kipp, S. Karakaya, J. Pawlak, G. Araujo-Wright, S. Arnold and C. Beyer, "Estrogen and the Development and Protection of Nigrostriatal Dopaminergic Neurons: Concerted Action of a Multitude of Signals, Protective Molecules, and Growth Factors," Frontiers in Neuroendocrinology, Vol. 27, No. 4, 2006, pp. 376-390. doi:10.1016/j.yfrne.2006.07.001

[25] E. Vegeto, V. Benedusi and A. Maggi, "Estrogen Anti-Inflammatory Activity in Brain: A Therapeutic Opportunity for Menopause and Neurodegenerative Diseases," Frontiers in Neuroendocrinology, Vol. 29, No. 4, 2008, pp. 507-519. doi:10.1016/j.yfrne.2008.04.001

[26] J. W. Simpkins and J. A. Dykens, "Mitochondrial Mechanisms of Estrogen Neuroprotection," Brain Research Reviews, Vol. 57, No. 2, 2008, pp. 421-430. doi:10.1016/j.brainresrev.2007.04.007

[27] J. D. Yager and J. Q. Chen, "Mitochondrial Estrogen Receptors-New Insights into Specific Functions,” Trends in Endocrinology \& Metabolism, Vol. 18, No. 3, 2007, pp. 89-91. doi:10.1016/j.tem.2007.02.006

[28] M. Cordey, U. Gundimeda, R. Gopalakrishna and C. J. Pike, "Estrogen Activates Protein Kinase C in Neurons: Role in Neuroprotection," Journal of Neurochemistry, Vol. 84, No. 6, 2003, pp. 1340-1348. doi:10.1046/j.1471-4159.2003.01631.x

[29] K. M. Dhandapani and D. W. Brann, "Role of Astrocytes in Estrogen-Mediated Neuroprotection," Experimental Gerontology, Vol. 42, No. 1-2, 2007, pp. 70-75. doi:10.1016/j.exger.2006.06.032

[30] C. L. Limoli, E. Giedsinski, H. Baure, R. Rola and J. R. Fike, "Redox Changes Induced Hippocampal Precursor Cells by Heavy Ion Irradiation," Radiation and Environmental Biophysics, Vol. 46, No. 2, 2007, pp. 167-172. doi:10.1007/s00411-006-0077-9

[31] R. Rola, V. Sarkissian, A. Obenhaus, G. A. Nelson, S. Otsuka, C. L. Limoli and J. R. Fike, "High-LET Radiation Induces Inflammation and Persistent Changes in Markers of Hippocampal Neurogenesis," Radiation Research, Vol. 164, No. 4, 2005, pp. 556-560. doi:10.1667/RR3412.1

[32] A. N. Carey, B. Shukitt-Hale, B. M Rabin and J. A. Joseph, "Interaction between Age and Exposure to ${ }^{56} \mathrm{Fe}$ Particles on Behavior and Neurochemistry," Advances in Space Research, Vol. 39, No. 6, 2007, pp. 987-993. doi:10.1016/j.asr.2006.11.012

[33] J. A. Joseph, W. A. Hunt, B. M. Rabin and T. K. Dalton, "Possible 'Accelerated Aging' Induced by ${ }^{56} \mathrm{Fe}$ Heavy Particle Irradiation: Implications for Manned Space Flights," Radiation Research, Vol. 130, No. 1, 1991, pp. 88-93. doi:10.2307/3578484

[34] J. A. Joseph, W. A. Hunt, B. M. Rabin, T. K. Dalton and A. H. Harris, "Deficits in Striatal Muscarinic Receptor Sensitivity Induced by ${ }^{56} \mathrm{Fe}$ Heavy Particle Irradiation: 
Further 'Age-Radiation' Parallels,” Radiation Research, Vol. 135, No. 2, 1993, pp. 257-261. doi:10.2307/3578303

[35] J. O. Strom, E. Theodorsson and A. Theodorsson, “Order of Magnitude Differences Between Methods for Maintaining Physiological 17B-Oestradiol Concentration in Ovariectomized Rats,” Scandinavian Journal of Clinical and Laboratory Investigation, Vol. 68, No. 8, 2008, pp. 814-822. doi:10.1080/00365510802409703

[36] B. M. Rabin, K. L. Carrihill-Knoll, M. Hinchman, B. Shukitt-Hale, J. A. Joseph and B. C. Foster, "Effects of Heavy Particle Irradiation and Diet on Object Recognition Memory in Rats," Advances in Space Research, Vol. 43, No. 8, 2009, pp. 1193-1199. doi:10.1016/j.asr.2009.01.015

[37] B. M. Rabin, J. A. Joseph and B. Shukitt-Hale, “A Longitudinal Study of Operant Responding in Rats Irradiated When 2 Months Old," Radiation Research, Vol. 164, No. 4, 2005, pp. 552-555. doi:10.1667/RR3349.1

[38] M. D. Lindner, M. A. Plone, J. M. Francis, T. J. Blane, J. D. Salmone and D. F. Emerich, "Rats with Partial Striatal Dopamine Depletions Exhibit Robust and Long-lasting Behavioral Deficits in a Simple Fixed-Ratio Bar Pressing Task," Behavioural Brain Research, Vol. 86, No. 1, 1997, pp. 25-40. doi:10.1016/S0166-4328(96)02240-1

[39] J. D. Salamone, "The Involvement of Nucleus Accumbens Dopamine in Appetitive and Aversive Motivation," Behavioural Brain Research, Vol. 61, No. 2, 1994, 117133. doi:10.1016/0166-4328(94)90153-8

[40] J. D. Salamone and M. Correa, "Motivational Reviews of Reinforcement: Implications for Understanding the Behavioral Functions of Nucleus Accumbens Dopamine,” Behavioural Brain Research, Vol. 137, No. 1-2, 2002, pp. 3-25. doi:10.1016/S0166-4328(02)00282-6
[41] G. Keppel, "Design and Analysis: A Researcher's Handbook," 4th Edition, Prentice Hall, Upper Saddle River, 1991.

[42] M. M. McCarthy, "How It's Made: Organisational Effects of Hormones on the Developing Brain," Journal of Neuroendocrinology, Vol. 22, No. 7, 2010, pp. 736-742. doi:10.1111/j.1365-2826.2010.02021.x

[43] T. Aubele, R. Kaufman, F. Montalmant and M. F. Kritzer, "Effects of Gonadectomy and Hormone Replacement on a Spontaneous Novel Object Recognition Task in Adult Male Rats,” Hormones and Behavior, Vol. 54, No. 2, 2008, pp. 244-252. doi:10.1016/j.yhbeh.2008.04.001

[44] R. B. Gibbs, "Testosterone and Estradiol Produce Different Effects on Cognitive Performance in Male Rats," Hormones and Behavior, Vol. 48, No. 3, 2005, pp. 266277. doi:10.1016/j.yhbeh.2005.03.005

[45] J. S. Sutcliffe, K. M. Marshall and J. C. Neill, "Influence of Gender on Working and Spatial Memory in the Novel Object Recognition Task in the Rat," Behavioural Brain Research, Vol. 177, No. 1, 2007, pp. 117-125. doi:10.1016/j.bbr.2006.10.029

[46] J. B. Becker, "Gender Differences in Dopaminergic Function in Striatum and Nucleus Accumbens," Pharmacology Biochemistry and Behavior, Vol. 64, No. 4, 1999, pp. 803-812. doi:10.1016/S0091-3057(99)00168-9

[47] D. Mitsushima, K. Takase, T. Funabashi and F. Kimura, "Gonadal Steroids Maintain 24 h Acetylcholine Release in the Hippocampus: Organizational and Activational Effects in Behaving Rats,” Journal of Neuroscience, Vol. 29, No. 12, 2009, pp. 3808-3815. doi:10.1523/JNEUROSCI.5301-08.2009 